\title{
Atypical Presentation of Lymphoma in an HIV-seropositive Child
}

\author{
AO Ogunseyinde*, JB Familusi**
}

\begin{abstract}
Summary
Ogunseyinde AO, Familusi JB. Atypical Presentation of Lymphoma in an HIVseropositive Child. Nigerian Journal of Paediatrics 2001;28:43. A case of lymphoma in an HIV sero-positive child whose parents also tested positive for the HIV infection is presented. He was initially being managed for meningitis in a private clinic. The rapid progression and dissemination of the disease to other parts of the body was no doubt, due to the HIV infection. It is important that health workers become more aware of the protean clinical manifestations of HIV infection in this environment where other childhood diseases share some of the clinical features and it should be considered in patients with lymphoma and rapid progression of the disease.
\end{abstract}

\section{Introduction}

ALTHOLIGH AIDS/HIV infection has reached epidemic proportions in sub-Saharan Africa, the clinical spectrum of the disease in affected individuals remains inadequately recognized. In Nigeria, most studies of HIV infection have concentrated on sero-prevalence, ${ }^{1,2}$ and the few clinical studies have tended to confine HIV screening to patients who satisfy the WHO clinical definition of AIDS. ${ }^{3,4}$ This has probably resulted in poor case recognition and underreporting in many sub-Saharan countries including Nigeria where the official cumulative number of AIDS cases in 1996 was only 0.03 per cent of the WHO estimates. ${ }^{5} \mathrm{~A}$ higher index of awareness is therefore required to determine the true prevalence of the disease in our environment. HIV is a retrovirus that attacks the $\mathrm{CD4}^{+} \mathrm{T}$ lymphocytes of the immune system, but can also infect several other types of cells in the body. In defence against the virus, the infected organism mounts an anti-viral response by production of $\mathrm{CD}^{+}$cell, a cytotoxic $\mathrm{T}$ lymphocyte response. HIV is a lenti-virus; it therefore, takes a long time for the clinical symptoms of the disease to manifest. Once activated, the virus replicates rapidly that treatment has to be given or the infection will rapidly progress to disease. To illustrate this point, we present the case of a previously well three and $a$ half-year old boy who had an aggressive course of illness due to HIV-associated lymphoma.

\section{University College Hospital, Ibadan}

\section{Department of Radiology}

* Reader

\section{Department of Paediatrics}

** Professor

Correspondence: $A O$ Ogunseyinde

\section{Case Report}

F was referred from a private clinic on September 11, 1997 with complaints of sudden onset of headaches and fever of two days' duration, followed by vomiting and swelling of the left eye of one day duration. He had been receiving intravenous penicillin and chloramphenicol for presumed meningitis in the private clinic.

The past medical history was unremarkable. He had had two episodes of upper respiratory tract infections, the last one occurring in January 1997. The prenatal history and delivery were normal and the developmental history to date was also normal. His parents were in good health, but his father had suffered from recurrent headaches in the preceding six months; the headaches were presumed to be psychosomatic because the onset followed the loss of his job. On examination, $F$ was well nourished and not overtly ill looking. He was not pale or jaundiced, but was moderately febrile (temperature, $38.5^{\circ} \mathrm{C}$ ). There was proptosis of the left eye, with associated dilated and unreactive left pupil. Pupillary reactions and vision were however, normal in the right eye. Fundoscopy of both eyes on admission revealed no abnormalities. Small discrete lymph nodes were palpable in both axillae and groins; the liver was palpable $2 \mathrm{~cm}$ below the right costal margin but the spleen was not palpable. The neck was supple and Kernig's sign was negative. A lumbar puncture done on admission, revealed normal cerebrospinal fluid. The chest radiograph revealed no abnormalities and tuberculin test was normal.

Five days after admission, the patient had become ill-looking and irritable; the proptosis had become bilateral but still remained more evident on the left side, and in addition to complete ophthalmoplegia of the left eye, the right eye now showed sixth cranial nerve paralysis and sluggish pupillary reaction. A computerized(CT) scan of the brain was then done because of suspected cavernous sinus thrombosis. This showed an enhancing mass in the naso-pharynx extending into the ethmoidal sinus and the medial aspect of both orbits 


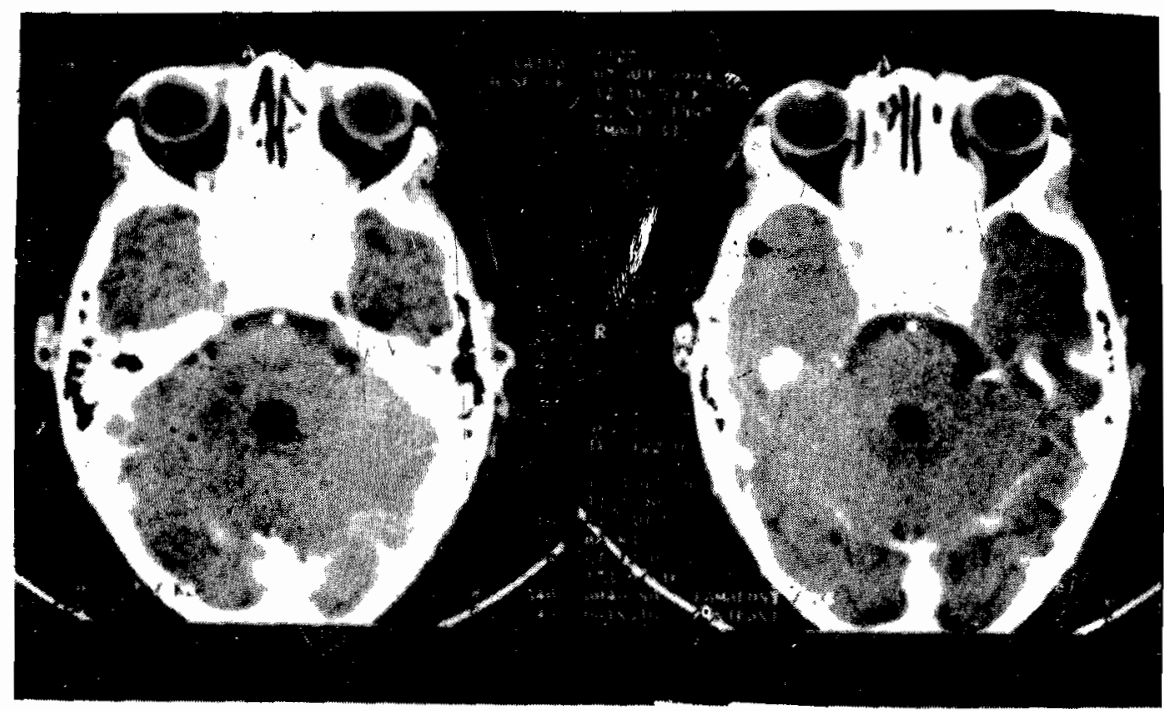

Fig. 1

Fig. 2

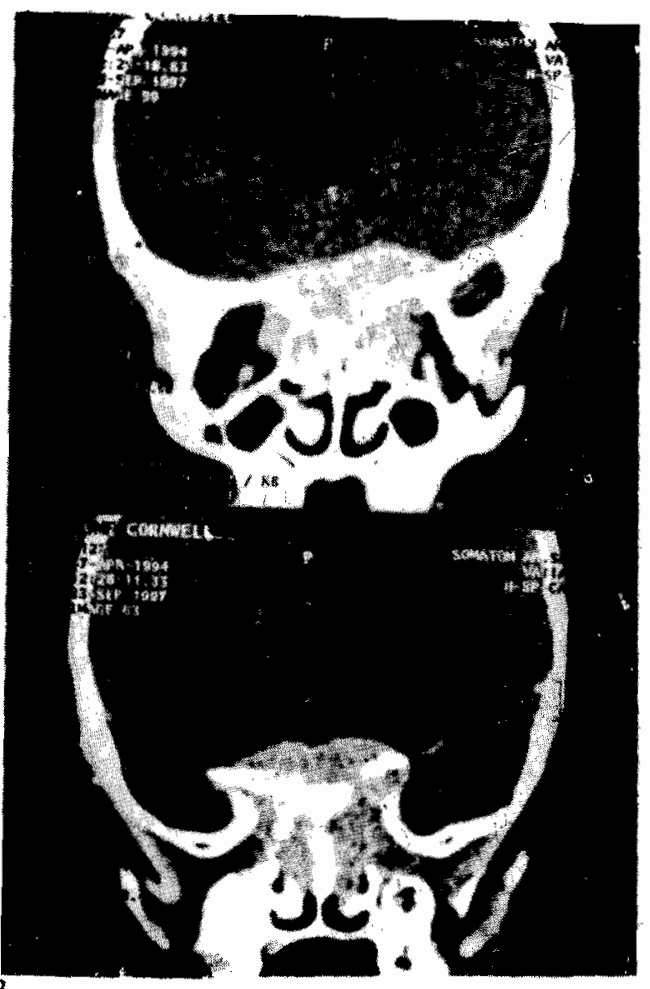

Contrast enhanced CT of the brain. Axial (Fig. 1) and coronal (Fig. 2) views showing a brilliantly enhancing mass in the base of skull anteriorly and extending into the orbits, ethmoidal sinuses and parasellar region.

with displacement of the medial rectus muscles. There was also opacification of the para-sella structures (Figs 1 and 2). A CT scan diagnosis of malignant disease, probably neuroblastoma or lymphoma, was suggested. The probability of HIV infection was considered at this point, and appropriate serological tests were ordered. Subsequent neurosurgical expiorauon and histological examination of the intracranial tumour biopsy revealed poorly differentiated small cell tumour considered to be either neuroblastomac $r$ lymphoma. The patient was then commenced on chemotherapy with vincristine, actinomycin Dand cyciophosphamide (VAC regi- men). His condition however did not improve, and three weeks into admission, a blood transfusion was given because of increasing anaemia.

On the twenty-second day of admission, he developed a swollen left leg; rectal and abdominal examinations at this time revealed poorly defined pelvic and abdominal masses confirmed by CT to be of mixed densities. The patient's condition continued to deteriorate and he died four days later. The results of the serological tests subsequently confirmed that he and his two parents were sero-positive for HIV infection. Detailed histological studies of the biopsy and autopsy materials confirmed a definitive diagnosis of non-Hodgkin's lymphoma.

\section{Discussion}

The age of our patient and the fact that his two' parents were sero-positive for HIV strongly suggest that his HIV infection resulted from mother to child transmission. Perinatal transmission is the most common route of HIV infection in children. ${ }^{6}$ About one-third of HIV-positive mothers are known to infect their offsprings and 80-90 per cent of all childhood infection are maternally transmitted. ${ }^{7,8}$ The known association between HIV infection and the development of malignancies (particularly lymphoma and Kaposi's sarcoma) in the African population ${ }^{9-11}$ also makes it likely that the non-Hodgkin lymphoma in our patient could be aetiologically related to his HIV infection although published report has suggested otherwise in Nigeria. ${ }^{3}$ This could be due to poor recognition and the attendant under-reporting. It is therefore important that health workers become more aware of the protean clinical manifestations of HIV infection in children and that essential diagnostic facilities become freely available.

The WHO ) definition of AIDS in children emphasized three major signs of weight loss or failure to thrive, chronic diarrhoea and prolonged fever, and six minor signs which include generalized lymphadenopathy, oro-pharyngeal candidiasis, persistent cough, generalized dermatitis, recurrent infections and confirmed maternal HIV infection. ${ }^{11,12} \mathrm{Pae}-$ 
diatric AIDS is to be suspected when a child presents with at least, two major signs associated with at least, two minor signs in the absence of a known cause of immuno-suppression. Our patient had only one minor sign namely generalized lymphadenopathy at presentation and no major sign. The subsequent confirmation of AIDS in him supports the opinion that the WHO clinical definition of paediatric AIDS lacks both sensitivity and specificity, particularly in our environment where other childhood diseases like tuberculosis, protein energy malnutrition, sickle cell anaemia and lymphoma which share some of the clinical features of AIDS are prevalent. ${ }^{7}$ Thus, a higher index of awareness than suggested by the WHO case definition is necessary for accurate diagnosis of the disease in the paediatric age group in our environment where the disease has now attained epidemic proportions. Studies in Central and Southern Africa ${ }^{12}$ have also found generalized lymphadenopathy to be the most common mode of presentation in children with symptomatic HIV infection. It seems reasonable therefore, to consider generalized lymphadenopathy to be a major (rather than a minor) AIDS defining sign in sub-Saharan children. The presonting features of headache, vomiting and ophthalmological signs suggested the probability of pathology of the nervous system which has been shown to occur in 30-50 per cent of $\mathrm{HIV}$ infected infants and children. ${ }^{13}$ These initial neurological signs and symptoms and subsequent rapid dissemination of disease to other areas of the body in our patient were no doubt due to the HIV associated lymphoma.

It is therefore important to consider the probability of HIV associated malignancy in any child presenting with sudden onset of neurological signs and symptoms especially as HIV is a multi-system disease.

\section{References}

1. Ministry of Health and Social Services. AIDS/HIV/STD control programme 1993/1994. Sentmel Seroprevalence Surveillance Report, 1995.

2. Olusanya $O$. Seroepidemiology of human retroviruses in Ogun State of Nigeria. Scand J Infect Dis 1990; 22:155-60.

3. Akinsete I, Akanmu AS, Okany CC. Spectrum of clinical díseases in HIV infected adults at the Lagos University Teaching Hospital: a five-year experience (1992-1996). Afr J Med med Sci 1998; 28: 147-51.

4. Angyo IA, Okpe ES, Onah J. Paediatric AIDS in Jos, Nigeria. West Afr J Med 1998; 17: 268-72.

5. WHO: AIDS-Global data, global situation of the HIV/AIDS pandemic. Wkly Epidemiol Rec 29 Nov. 1996: 361-4.

6. WoodWP. Children with acquired immune deficiency syndrome. Invest Radiol 1992; 27:964-70.

7. Vetter KM, Djomand G, Zadi F et al. Clinical spectrum of human immuno-deficiency vir us disease in children in a West African city. Pediatr Infect Dis J1996; $15: 438-42$.

8 Falcon R, Eddy J, Wiener I, Pizzo P. Immuno-deficient virus infection in children. J Pediatrics 1989; 114: 1-30.

9 Lucas SB, Diamande M, Hounnou A, Beaumel A et al. HIV associated lymphoma in Africa: an autopsy study in Cote D'Ivoire. lnt J Cancer 1994; 59: 20-4.

10. Sitas F, BezwodaWR, Levin V, RuffP et al. Association between human immunodeficiency virus type 1 infection and cancer in black population of Johannesburg and Soweto, South Africa. Brit/ Cancer 1997; 75 : 1704-7.

11. Meerstadt S, Brueton MJ. Paediatric AIDS. Postyrad Doctor (Africa) 1987; 9:234-40.

12.Coovadia HM, Wittenberg DF. HIV infection. In:Coovadia HM, Wittenberg DF, eds. Paediatrics and Child Health. Capetown: Oxford University Press (Publishers) Ltd, 1998:3419.

13.Belman Al, Diamond D, Dickson D, et al . Paediatric acquired immunodeficiency syndromes. Am J Dis Child 1998; $152: 29$. 35. 